\title{
Filling the Gap 115 Years after Ronald Ross: The Distribution of the Anopheles coluzzii and Anopheles gambiae s.s from Freetown and Monrovia, West Africa
}

\author{
Dziedzom K. de Souza', Benjamin G. Koudou, ${ }^{2,4}$, Fatorma K. Bolay ${ }^{3}$, Daniel A. Boakye', \\ Moses J. Bockarie ${ }^{2 *}$
}

1 Parasitology Department, Noguchi Memorial Institute for Medical Research, University of Ghana, Accra, Ghana, 2 Centre for Neglected Tropical Diseases, Liverpool School of Tropical Medicine, Liverpool, United Kingdom, 3 Liberian Institute for Biomedical Research, Charlesville, Liberia, 4 Centre Suisse de Recherches Scientifiques en Cote d'Ivoire, Abidjan, Cote d'Ivoire

\begin{abstract}
It was in Freetown, Sierra Leone, that the malaria mosquito Anopheles coastalis, now known as Anopheles gambiae, was first discovered as the vector of malaria, in 1899. That discovery led to a pioneering vector research in Sierra Leone and neighbouring Liberia, where mosquito species were extensively characterized. Unfortunately, the decade long civil conflicts of the 1990s, in both countries, resulted in a stagnation of the once vibrant research on disease vectors. This paper attempts to fill in some of the gaps on what is now known of the distribution of the sibling species of the An. gambiae complex, and especially the An. coluzzii and An. gambiae s.s, formerly known as the An. gambiae molecular M and S forms respectively, in the cities of Freetown and Monrovia.
\end{abstract}

Citation: de Souza DK, Koudou BG, Bolay FK, Boakye DA, Bockarie MJ (2013) Filling the Gap 115 Years after Ronald Ross: The Distribution of the Anopheles coluzzii and Anopheles gambiae s.s from Freetown and Monrovia, West Africa. PLoS ONE 8(5): e64939. doi:10.1371/journal.pone.0064939

Editor: Georges Snounou, Université Pierre et Marie Curie, France

Received March 19, 2013; Accepted April 23, 2013; Published May 31, 2013

Copyright: (c) 2013 de Souza et al. This is an open-access article distributed under the terms of the Creative Commons Attribution License, which permits unrestricted use, distribution, and reproduction in any medium, provided the original author and source are credited.

Funding: This study was supported by the Centre for Neglected Tropical Diseases, Liverpool School of Tropical Medicine, through funding from DFID and GSK. The funders however played no role in the publication of this manuscript. The funders had no role in study design, data collection and analysis, decision to publish, or preparation of the manuscript

Competing Interests: This study was supported by the Centre for Neglected Tropical Diseases, Liverpool School of Tropical Medicine, through funding from DFID and partly from GSK. There are no patents, products in development or marketed products to declare. This does not alter the authors' adherence to all the PLOS ONE policies on sharing data and materials, as detailed online in the guide for authors.

* E-mail: Moses.Bockarie@liverpool.ac.uk

\section{Introduction}

Anopheles gambiae (formally known An. coastalis) was first incriminated as a vector of malaria by Sir Ronald Ross in Freetown, Sierra Leone in 1899 [1]. Mosquitoes belonging to the Anopheles gambiae complex are the major vectors of malaria in West Africa. As such studying their distribution, ecology and population structure is essential for effective malaria control programs. In the majority of West African countries, various studies on these important vectors have been undertaken in details. However, there is a gap in knowledge of these vectors in post conflict countries such as Sierra Leone and Liberia where ecological studies could not be undertaken in the 1990s and early 2000s because of the civil war that engulfed both countries for more than a decade.

Prior to the civil unrest in these countries, the mosquito species and their distribution were largely documented. Sierra Leone primarily served as the centre of malaria vector research and control, being the place where human malarial parasites were first observed in wild-caught An. gambiae and An. funestus, in 1899 [1]. By the 1950s extensive studies on human biting mosquitoes in both Sierra Leone [1] and Liberia [2-5] had been undertaken. However, these studies were focussed on the morphological identification of these important vectors.

By the 1980s the study of An. gambiae vectors of malaria in West Africa had evolved from morphometric to cytotaxonomic studies $[6,7]$. The development of the PCR in 1990 [8] led to a further evolution in the identification of the sibling species of the $A n$. gambiae complex [9], the $\mathrm{M}$ and $\mathrm{S}$ molecular forms of the An. gambiae s.s. [10] and knockdown resistance markers to insecticides $[11,12]$ using molecular methods. Following molecular and bionomical evidence, the An. gambiae molecular $\mathrm{M}$ form has subsequently been renamed An. coluzzii, whiles the An. gambiae molecular S form retained the name An. gambiae s.s [13]. These studies have been undertaken and enhanced malaria control activities in most countries in West Africa. Unfortunately, the civil conflict in Liberia (1989-1996, 1999-2003) and Sierra Leone (1991-2002) stagnated the once vibrant malaria vector research activities in the cities of Monrovia and Freetown. To date, no molecular identification of members of the An. gambiae complex had been undertaken/published in Sierra Leone, and in Liberia only one study [14] has described the distribution of the $\mathrm{M}$ and $\mathrm{S}$ forms of the An. gambiae s.s and their $k d r$ frequencies in the Bomi County. In this short communication, we report the distribution of the An. coluzzii and the An. gambiae s.s., in Freetown and Monrovia the capital cities of Sierra Leone and Liberia respectively.

\section{Methods}

\section{Study Sites}

In Sierra Leone, samples were collected in November-December 2009, from 89 households in 5 communities around Freetown. In Liberia, samples were collected in July 2011, from 100 

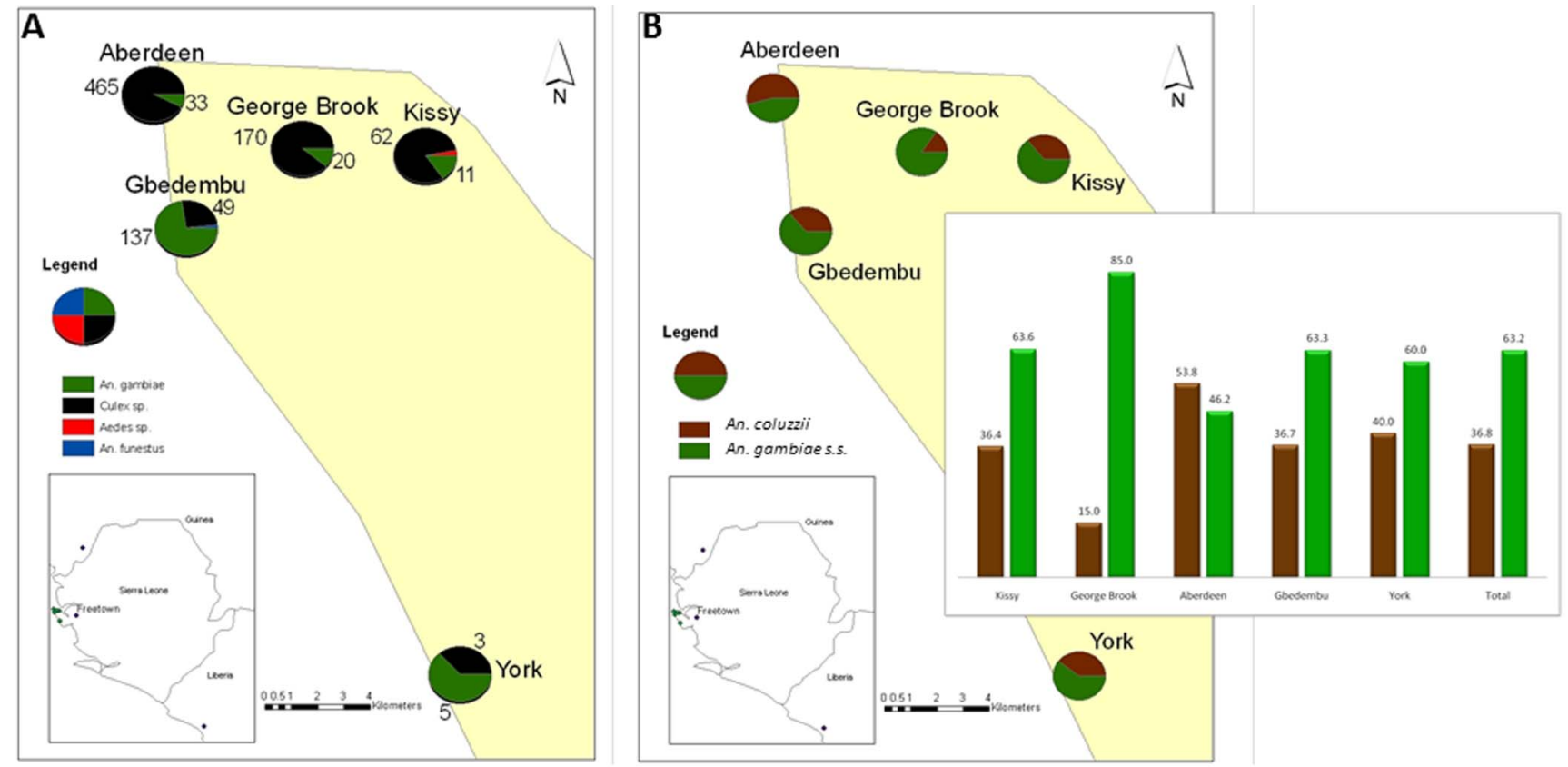

Figure 1. Distribution of the mosquito species, the An. coluzzii and the An. gambiae s.s from the sample communities in Freetown, Sierra Leone.

doi:10.1371/journal.pone.0064939.g001

households in 4 communities in Monrovia (See Figures 1 and 2 below). Samples were collected using the pyrethrum spray method. In each community, samples were collected early in the morning between 5 and 9 am, in different households, over a 3 days period.

The collected samples were identified morphologically and the Anopheles species were stored individually on silica gel, while the Culex spp. specimens were put together in pools of 20. Other mosquito species were also stored separately. The collected samples were brought to the Noguchi Memorial Institute for Medical Research, Ghana, for molecular identification.

\section{Molecular Studies}

Genomic DNA was extracted from 2-3 legs from each mosquito, using the boil preparation method. Briefly, the legs were crushed in $100 \mu \mathrm{l}$ of distilled water and boiled at $95^{\circ} \mathrm{C}$ for 10 minutes. The supernatant was used as template for the PCR. The extracted DNA was used for species identification using Polymerase Chain Reaction, Restriction Fragment Length Polymorphism (PCR-RFLP) [10]. The determination of the knockdown mutation conferring resistance to pyrethroids was undertaken using the method of Martinez-Torres et al [11].

\section{Ethics Statement}

Approval for this study was obtained from the IRB of the Liverpool School of Tropical Medicine and the Ethics and Scientific Review Committees of the Ministries of Health in Sierra Leone and Liberia. The communities, where sampling was done, were informed on the project and consent sought from the local authorities within each community. Consent was also sought from the households where mosquito sampling was carried out.

\section{Results and Discussion}

\section{Specimen Collection and Morphological Identification}

The number of collections from each community in Freetown is shown in Table 1. Overall, 206 An. gambiae and 749 Culex species were collected. In Monrovia, 161 and 381 An. gambiae and Culex species were collected respectively. Culex was the most common mosquito species collected, due to the polluted breeding waters found in the areas where the collections were made. An. funestus and Aedes spp. were also collected, but in very low numbers. The distribution of the mosquito species collected in Freetown and Monrovia is shown in Figure 1A and 2A respectively.

\section{Molecular Identification and Distribution of the An. coluzzii and An. gambiae s.s}

194 samples were analyzed for the molecular identification of the members of the An. gambiae complex in Freetown. 182 (93.8\%) were successfully identified as either An. coluzzii or An. gambiae s.s. The remaining 12 samples failed to amplify. Further differentiation revealed $63.2 \%$ of the samples analyzed to be An. gambiae s.s., while the remaining $36.8 \%$ were $A n$. coluzzii. The distribution of An. gambiae s.s. and An. coluzzii is shown in Figure 1B below. This study is the first to analyze the distribution of the An. gambiae s.s. and An. coluzzii in Freetown, Sierra Leone. Sierra Leone is a mountainous, forested region and this may explain the high proportion of $A n$. gambiae s.s. compared to An. coluzzii, and is in accordance with a study [15] that showed that the An. gambiae molecular S form was dominant in mountainous, forested areas.

In Monrovia, 105 mosquitoes morphologically identified as $A n$. gambiae were analyzed. Out of these, $90.5 \%$ were identified as $A n$. coluzzii, and the remaining $9.5 \%$ as An. gambiae s.s. These results are consistent with the results of Temu and colleagues [14] which showed $98 \%$ of samples identified to be the An. gambiae molecular $\mathrm{M}$ form in the Bomi County. Figure 2B shows the distribution of An. gambiae s.s. and An. coluzzii in the collection sites in Monrovia. 

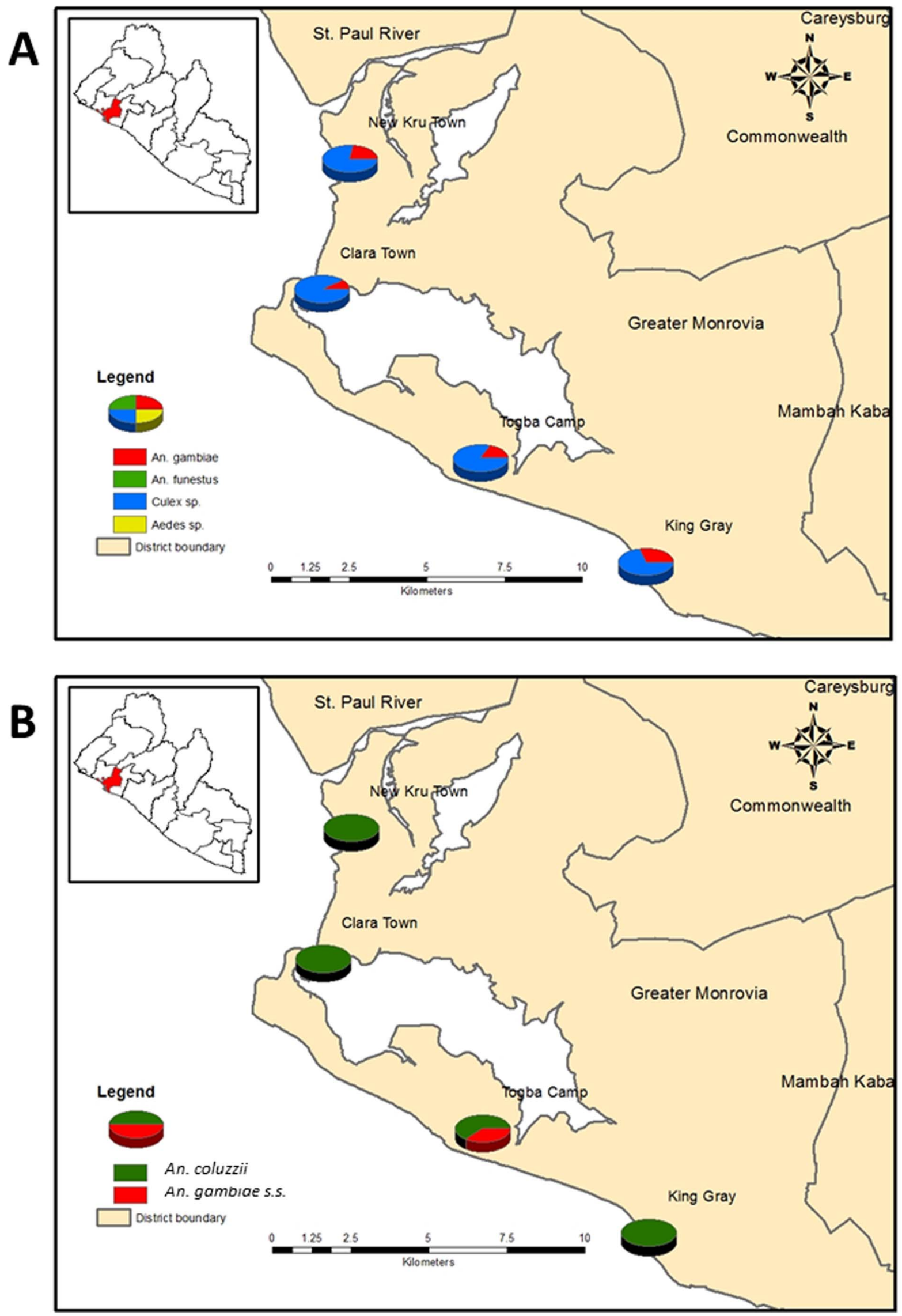
Figure 2. Distribution of the mosquito species, the $A n$. coluzzii and the An. gambiae s.s from the sample communities in Monrovia, Liberia.

doi:10.1371/journal.pone.0064939.g002

\section{Distribution of Knockdown Resistance Mutations}

Very high proportions of $k d r$ mutations $(96.2 \%)$ were detected in the An. gambiae populations (Table 2). The mutation was present both in An. gambiae s.s. and An. coluzzii, but was more common in the An. gambiae s.s. Few hybrids were also detected. The presence of the $k d r$ mutation was initially thought to be only present in the $A n$. gambiae molecular $\mathrm{S}$ form. However, studies have shown its occurrence in the An. gambiae molecular M form [16-20]. This study showed the mutation in $91 \%$ of the An. coluzzii identified, similar to values recently reported in the An. gambiae molecular M form in Cote d'Ivoire [20]. It is suggested that the presence of the mutation in the $\mathrm{M}$ form may be a result of introgression from the $\mathrm{S}$ form [17]. However, observations from the Island of BiokoEquatorial Guinea, where the mutation was only observed in the $\mathrm{M}$ form [19], suggested that the mutation in the $\mathbf{M}$ form may have arisen independently due to intensive insecticide application.

The very high levels of $k d r$ resistance to pyrethroids might be due to the high usage of insecticides (e.g. BHC and DDT) for malaria control activities in Sierra Leone, with possible cross resistance to pyrethroids. Studies conducted in the late 1950's, on the development of insecticide resistance in the An. gambiae in Freetown, showed the factor conferring resistance to $\mathrm{BHC}$ in $66 \%$ of the population [21]. Dieldrin resistance was observed in 91$100 \%$ of An. gambiae populations in neighboring Liberia in 1957 [22] and was reported in Freetown in 1966 [23]. HCH and DDT were widely used for house spraying and larviciding in Freetown after the World War II [1]. Temephos and malathion were also used for larviciding until it was realized that control activities were unsuccessful, with a possible resistance to malathion [1]. Despite these reports we cannot conclusively assign the observed high levels of $k d r$ only to insecticide use in the 1950's and further studies will be required to assess this.

While the frequencies of $k d r$ mutations were not determined for the Monrovia samples, it is believed that the situation may not be much different from Freetown, considering the mosquito control activities that were in place in the 1950s $[1,24]$. Also, recent studies in the Bomi County revealed $k d r$ frequencies of over $90 \%$ [14].

Table 1. Number of mosquito collections from each community in Freetown, Sierra Leone.

\begin{tabular}{|c|c|c|c|c|c|}
\hline Country & Community & An. gambiae & Culex sp. & Aedes sp. & An. funestus \\
\hline \multirow{6}{*}{$\begin{array}{l}\text { Sierra } \\
\text { Leone }\end{array}$} & Kissy & 11 & 62 & 2 & - \\
\hline & George Brook & 20 & 170 & - & - \\
\hline & Aberdeen & 33 & 465 & - & - \\
\hline & Gbedembu & 137 & 49 & - & 3 \\
\hline & York & 5 & 3 & - & - \\
\hline & Total & 206 & 749 & 2 & 3 \\
\hline \multirow[t]{5}{*}{ Liberia } & Clara Town & 25 & 70 & 3 & - \\
\hline & New Kru Town & 43 & 72 & - & - \\
\hline & King Gray & 61 & 89 & - & - \\
\hline & Togba Camp & 32 & 87 & - & 1 \\
\hline & Total & 161 & 318 & 3 & 1 \\
\hline
\end{tabular}

Table 2. The distribution of the $k d r$ allele in the An. coluzzii and the An. gambiae s.s in Freetown.

\begin{tabular}{|c|c|c|c|c|}
\hline \multirow[b]{2}{*}{ Communities } & \multirow[b]{2}{*}{ An. spp } & \multicolumn{3}{|l|}{$k d r$ Allele } \\
\hline & & $\mathbf{R R}$ & SS & RS \\
\hline \multirow[t]{2}{*}{ Aberdeen } & An. coluzzii & $85.7 \%(12)$ & $14.3 \%(2)$ & - \\
\hline & An. gambiae s.s & $100 \%(12)$ & - & - \\
\hline \multirow[t]{2}{*}{ Gbedembu } & An. coluzzii & $93.2 \%(41)$ & $6.8 \%(3)$ & - \\
\hline & An. gambiae s.s & $97.4 \%(74)$ & - & $2.6 \%(2)$ \\
\hline \multirow[t]{2}{*}{ George Brook } & An. coluzzii & $66.7 \%(2)$ & $33.3 \%(1)$ & \\
\hline & An. gambiae s.s & $88.2 \%(15)$ & $5.9 \%(1)$ & $5.9 \%(1)$ \\
\hline \multirow[t]{2}{*}{ Kissi } & An. coluzzii & $100 \%(4)$ & - & - \\
\hline & An. gambiae s.s & $100 \%(7)$ & - & - \\
\hline \multirow[t]{2}{*}{ York } & An. coluzzii & $100 \%(2)$ & - & - \\
\hline & An. gambiae s.s & $100 \%(3)$ & - & - \\
\hline \multirow[t]{2}{*}{ Total } & An. coluzzii & $91.1 \%(61)$ & $8.9 \%(6)$ & - \\
\hline & An. gambiae s.s & $96.5 \%(111)$ & $0.9 \%(1)$ & $2.6 \%(3)$ \\
\hline
\end{tabular}

doi:10.1371/journal.pone.0064939.t002

Although these results may represent initial information, it was recently demonstrated that there is no significant association between the presence of the $1014 \mathrm{~F} k d r$ allele and ability to survive exposure to pyrethroid [20]. Thus insecticide susceptibility testing and bio-assay data are necessary to validate our findings.

\section{Conclusions}

The distribution of the An. coluzzii and An. gambiae s.s and prevalence of the $k d r$ gene are described here for the first time in Sierra Leone, nearly 115 years after the incrimination of the Anopheles mosquito as the vector of malaria. This study showed that the An. gambiae s.s is the most dominant sibling species of $A n$. gambiae complex in Freetown, while the An. coluzzii is the dominant species in Monrovia. A high prevalence of $k d r$ mutation has been observed in both An. coluzzii and An. gambiae s.s in Freetown. There is however a limitation to these results, in that the determination of the $k d r$ frequencies was not undertaken on surviving or dead mosquitoes exposed to pyrethroids through insecticide susceptibility testing. However, due to the high levels of $k d r$ mutation in the An. coluzzii and An. gambiae s.s populations, it is recommended that insecticide susceptibility testing be done on non-pyrethroids insecticides (organophosphates and carbamates) in order to determine the insecticide of choice for future vector control activities.

\section{Acknowledgments}

We would like to acknowledge the support of the study communities, individuals and field/research assistants who helped in undertaking this study.

\section{Author Contributions}

Conceived and designed the experiments: DKdS BGK FKB DAB MJB. Performed the experiments: DKdS. Analyzed the data: DKdS BGK MJB. Contributed reagents/materials/analysis tools: FKB DAB MJB. Wrote the paper: DKdS BGK FKB DAB MJB. 


\section{References}

1. Bockarie MJ, Gbakima AA, Barnish G (1999) It all began with Ronald Ross: 100 years of malaria research and control in Sierra Leone (1899-1999). Ann Trop Med Parasitol 93: 213-224.

2. Bequaert J (1930) Medical and economic entomology. Culicidae. In: Strong R, editor. The African republic of Liberia and the Belgian Congo-Harvard Africa Expedition 1926-27. Cambridge: Harvard University Press. 825-846.

3. Briscoe M (1947) Insect reconnaissance in Liberia, West Africa. Psyche 54: 245255.

4. Briscoe M (1950) Field notes on mosquitoes collected in Liberia, West Africa. Mosquito News 10: 19-21.

5. Briscoe M (1952) The relations of insects and insectborne diseases to the vegetation and environment in Liberia. Ecology 33: 187-214.

6. Coluzzi M, Petrarca V, Di Deco MA (1985) Chromosomal inversion intergradation and incipient speciation in Anopheles gambiae. Bollettino di Zoologia 52: 45-63.

7. Coluzzi M, Sabatini A, Petrarca V, Di Deco MA (1979) Chromosomal differentiation and adaptation to human environments in the Anopheles gambiae complex. Trans R Soc Trop Med Hyg 73: 483-497.

8. Mullis KB (1990) The unusual origin of the polymerase chain reaction. Scientific American 262: 56-65.

9. Scott JA, Brogdon WG, Collins FH (1993) Identification of single specimens of the Anopheles gambiae complex by the polymerase chain reaction. Am J Trop Med Hyg 49: 520-529.

10. Favia G, della Torre TA, Bagayoko M, Lanfrancotti A, Sagnon N, et al. (1997) Molecular identification of sympatric chromosomal forms of Anopheles gambiae and further evidence of their reproductive isolation. Insect Mol Biol 6: 377-383.

11. Martinez-Torres D, Chandre F, Williamson MS, Darriet F, Berge JB, et al. (1998) Molecular characterization of pyrethroid knockdown resistance $(k d r)$ in the major malaria vector Anopheles gambiae s.s. Insect Mol Biol 7: 179-184.

12. Gentile G, Santolamazza F, Fanello C, Petrarca V, Caccone A, et al. (2004) Variation in an intron sequence of the voltage-gated sodium channel gene correlates with genetic differentiation between Anopheles gambiae s.s. molecular forms. Insect Mol Biol 13: 371-377.
13. Coetzee M, Hunt RH, Wilkerson R, Della Torre A, Coulibaly MB, et al. (2013) Anopheles coluzzii and Anopheles amharicus, new members of the Anopheles gambiae complex. Zootaxa 3619: 246-274.

14. Temu E, Maxwell C, Munyekenye G, Howard A, Munga S, et al. (2012 Pyrethroid Resistance in Anopheles gambiae, in Bomi County, Liberia, Compromises Malaria Vector Control. PLoS One 7: e44986.

15. de Souza D, Kelly-Hope L, Lawson B, Wilson M, Boakye D (2010) Environmental Factors Associated with the Distribution of Anopheles gambiae s.s in Ghana; an Important Vector of Lymphatic Filariasis and Malaria. PLoS One 5: e9927.

16. Diabate A, Brengues C, Baldet T, Dabire KR, Hougard JM, et al. (2004) The spread of the Leu-Phe $k d r$ mutation through Anopheles gambiae complex in Burkina Faso: genetic introgression and de novo phenomena. Trop Med Int Health 9: 1267-1273.

17. Weill M, Chandre F, Brengues C, Manguin S, Akogbeto M, et al. (2000) The $k d r$ mutation occurs in the Mopti form of Anopheles gambiae s.s. through introgression. Insect Mol Biol 9: 451-455.

18. Yawson AE, McCall PJ, Wilson MD, Donnelly MJ (2004) Species abundance and insecticide resistance of Anopheles gambiae in selected areas of Ghana and Burkina Faso. Med Vet Entomol 18: 372-377.

19. Reimer LJ, Tripet F, Slotman MA, Spielman A, Fondjo E, et al. (2005) An unusual distribution of the $k d r$ gene among populations of Anopheles gambiae on the island of Bioko, Equatorial Guinea. Insect Mol Biol 14: 683-688.

20. Edi CVA, Koudou BG, Jones CM, Weetman D, Ranson H (2012) Multiple insecticide resistance in Anopheles gambiae mosquitoes, Southern Côte d'Ivoire. Emerg Infect Dis 18: 1508-1511.

21. Micks DW (1960) Insecticide-Resistance; A Review of Developments in 1958 and 1959. Bull Wld Hlth Org 22: 519-529.

22. Brown AWA (1958) The Insectice-Resistance Problem; A Review of Developments in 1956 and 1957. Bull Wld Hlth Org 18: 309-321.

23. Storey J (1967) Assignment Report: Malaria Pre-eradication Programme. Freetown. Document AFR/MAL/77Geneva: World Health Organization.

24. Poindexter H (1950) Filariasis bancrofti studies in Liberia. Am J Trop Med Hyg 30: 519-523. 